\title{
Chemically Converted Graphene as a Hole Transport Layer (HTL) Inorganic Photovoltaics (OPVS)
}

\author{
Farjana Haque ${ }^{1}$, Md. Moshiur Rahman ${ }^{2}$, Md. Abdullah Al Mahmud ${ }^{3 *}$, \\ M. Subbir Reza ${ }^{4}$, Munmun Akter ${ }^{5}$, A.H.M Zadidul Karim ${ }^{6}$
}

${ }^{1}$ University of Dhaka, BANGLADESH

2,3,4,5,6 University of Asia Pacific (UAP), BANGLADESH

*Corresponding Contact:

Email: mdabdullahalmahmud123@gmail.com

\begin{abstract}
Concerns about Global Warming and diminishing fossil fuel reserves have accelerated the search for low cost sources of renewable energy. Organic photovoltaics (OPVs) could be one such source; however, they have a list of shortcomings, including low efficiencies, short lifetimes, and reliance on poly (3, 4-ethylenedioxythiophene) polystyrene sulfonate (PEDOT: PSS), an expensive and highly acidic $(\mathrm{pH}=1)$ hole transport layer. Replacing PEDOT: PSS with chemically derived graphene may eliminate one of the drawbacks associated with OPVs. This paper took the first step towards that goal by developing a process to synthesize and characterize inverted and normal poly (3-hexylthiophene) (P3HT), [6, 6]phenyl-C61 butyric acid methyl ester (PCBM) solar cells. Although detrimental to the stability of the cells, ambient synthesis replicated the conditions required for large-scale, industrial production. The utilization of graphene oxide (GO) thin films as the hole transport and electron blocking layer in organic photovoltaics (OPVs) is demonstrated. The incorporation of GO deposited from neutral solutions between the photoactive poly(3-hexylthiophene) (P3HT):phenyl-C61 butyric acid methyl ester (PCBM) layer and the transparent and conducting indium tin oxide (ITO) leads to a decrease in recombination of electrons and holes and leakage currents. This results in a dramatic increase in the OPV efficiencies to values that are comparable to devices fabricated with PEDOT: PSS as the hole transport layer. Our results indicate that GO could be a simple solution process able Alternative to PEDOT: PSS as the effective hole transport and electron blocking layer in OPV and light-emitting diode devices.
\end{abstract}

\section{Key words}

Organic photovoltaics (OPVs), Graphene oxide (GO), poly (3, 4-ethylenedioxythiophene) polystyrene sulfonate 


\section{INTRODUCTION}

In the present paper, we have investigated chemically derived graphene oxide-based thin films as anode interfacial layers for high performance and stable polymer solar cells. We have deposited the hole transport layer(HTL) of grapheme oxide on top of the transparent and conducting ITO (indium tin oxide) anode, the third layer is the active layer of blends of P3HT:PCBM and the forth one is the $\mathrm{Al}$ cathode. Among various methods the chemical vapor deposition (CVD) is scalable for wrinkle-free, large-area graphene film. However, the preparation conditions and procedure are complex and it allows to grow GO only on metals (Wang et al., 2012). The as-prepared GO films are electrically insulating due to the heavy oxygenation of graphene sheets but could be made conductive by annealing into reduced graphene oxide (rGO) to facilitate the carrier transport more efficiently. The conductivity of GO film is promoted due to increased percolation through the film by removal of oxidized functionalities. rGO films have been prepared either chemically or thermally. As thermal reduction has been performed only at high temperatures $\left(400-1100{ }^{\circ} \mathrm{C}\right)$ a low processing temperature is highly desirable for making it useful. Annealing temperature as low as $170^{\circ} \mathrm{C}$ for 1 hour was assessed to be the optimum percolation condition of GO films, which makes this process appealing for practical applications. To make a low cost solution processable and roll to roll processed OSCs, we have used developed cost efficient and simply processable graphene oxide as interfacial materials and have done various experiments and plotted several responses to come to know the efficiency and other aspects of our cell.

\section{EXPERIMENTAL SetUP}

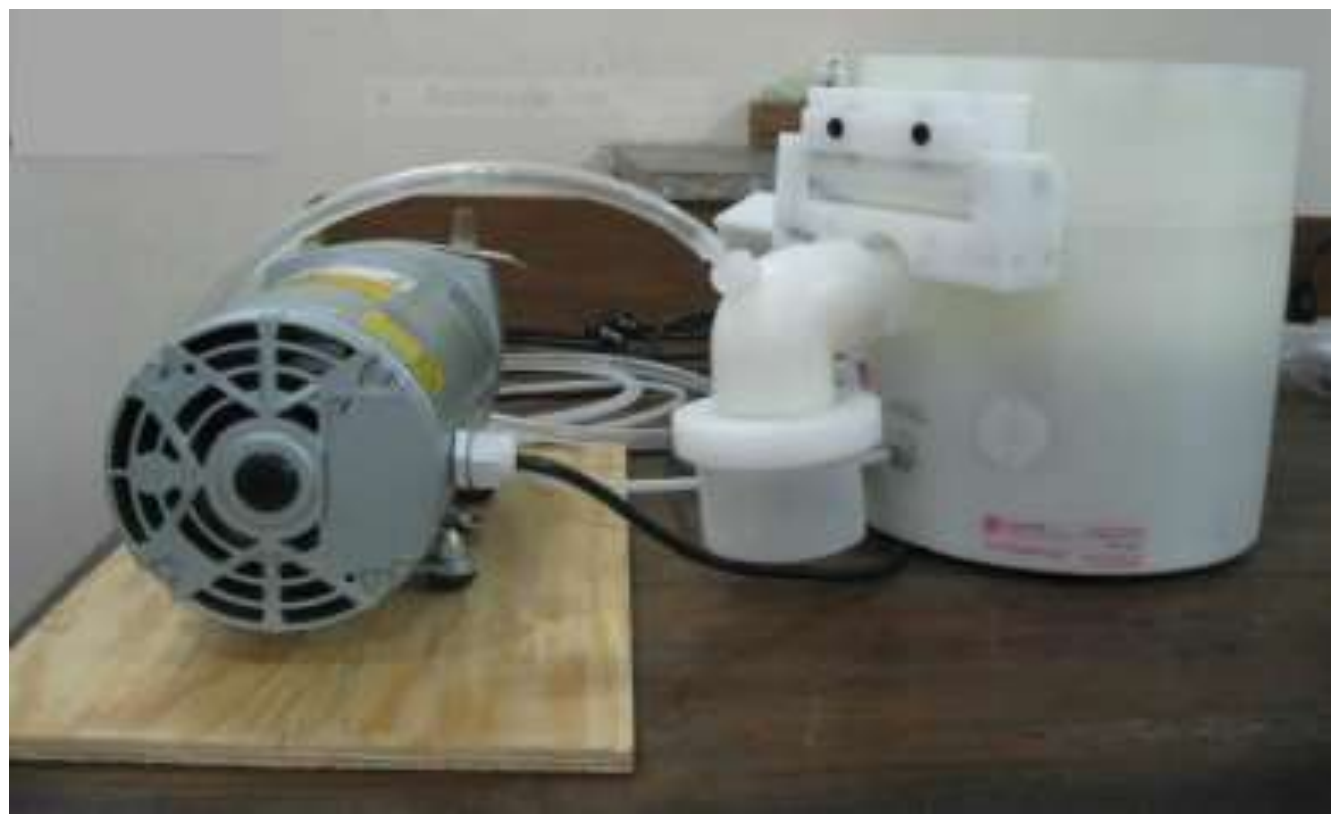

Figure 1: Photograph of a spin coater.

The designed ITO substrate was purchased from ossila, UK. After ultrasonic at on the GO solution of concentration of $1 \mathrm{mg} / \mathrm{mL}$ was deposited in the middle of the ITO slide using a micropipette. The GO was spin coated at $3400 \mathrm{rpm}$ for 30 seconds with the spin coater. The hole transport layer (GO solution) was $20 \mathrm{~nm}$ thick. The GO layer was annealed at 90-100 ${ }^{\circ} \mathrm{C}$ for 20 minutes. 


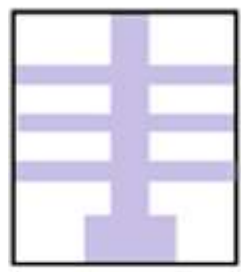

(a)

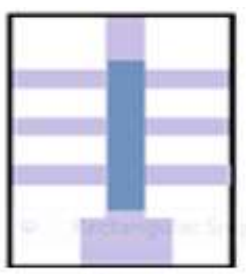

(b)

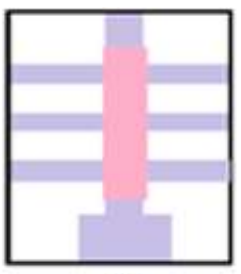

(c)

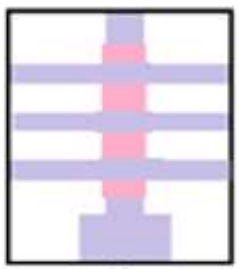

(d)

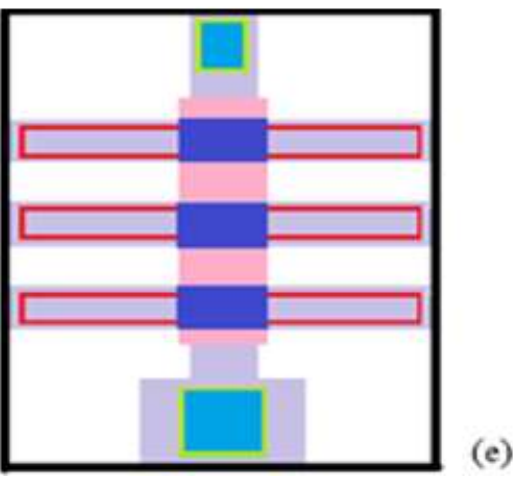

Area of glass substrate

Back electrode(Al)

Contact with front electrode (ITO)

Area of a single cell(total 3 cells)

Figure 2: Steps for fabrication of a normal geometry cell: a) the pre-patterned ITO substrate b) the substrate after GO was spin coated and wiped out of the ITO stripes and areas that would serve as points of contact with the ITO front electrode c) the substrate after spin coating the P3HT:PCBM and wiping out the excess active material d) the substrate after Al evaporation e) a substrate that is labeled to show the three cells contained on the substrate as well as the contact points for the front and back electrodes.

After stirring overnight, the active layer blend P3HT: PCBM solution in triclorobenzene with a concentration of $20 \mathrm{mg} / \mathrm{mL}$ was deposited in the middle of the slide, which was spun at $1500 \mathrm{rpm}$ for $45 \mathrm{~s}$. Excess active material was wiped off using Chlorobenzene-soaked Qtips. The slide was annealed at $150{ }^{\circ} \mathrm{C}$ for 10 minutes. As cathode an $\mathrm{Al}$ electrode was evaporated (vacuum evaporation) following the annealing period. The cell was placed active material down into the machined mask making sure to align the ITO patches with the correct openings in the musk. After evaporation the cell was allowed to cool in the evaporator for 30 minutes. As the electrodes, $\mathrm{Cu}$ wire was connected to the cell with the help of silver paste.

\section{Solar cells characterization}

Measuring a solar cell's current as a function of voltage and generating IV curves (see Figure 3) provides most of the information necessary to characterize a solar cell. An ideal solar cell operates like a diode in parallel with a current source. When no light shines on the cell, the IV curve resembles the IV curve of a diode (Figure 3). The absorption of light generates a current, and since power is given by,

$\mathrm{P}=\mathrm{VI}$

Where, I is current and V is voltage, the solar cell's maximum power can be derived from the IV curve.

A solar cell's performance is also evaluated by calculating the fill factor, a ratio of maximum measured power to maximum theoretical power. The equation for fill factor (FF) is given by, 
$\mathrm{FF}=\frac{\operatorname{Imax} \operatorname{Vmax}}{\text { Isc Voc }}$

where ISC is the short circuit current (current when voltage equals zero), and VOC is the open circuit voltage (voltage with zero current).

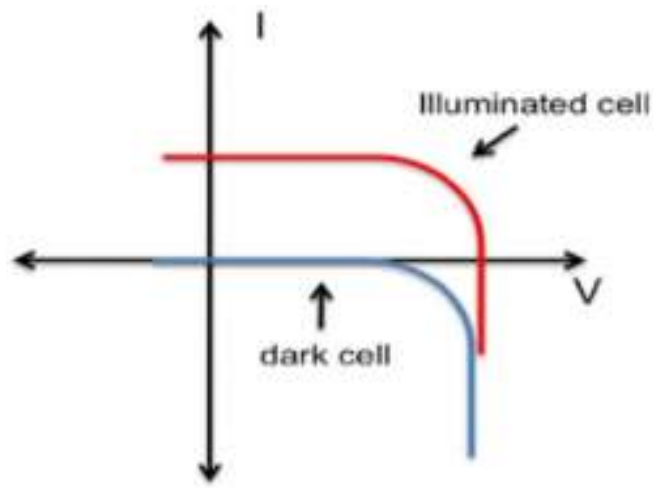

Figure 3: The IV curves of an illuminated and dark solar cell; adapted from [2].

Modeling a solar cell as a circuit, in this case a diode and current source in parallel and in series with resistors provides additional information in parallel and in series with resistors, provides additional information about the cell (see Figure 5). The equation for current of this circuit is given by,

$I=I_{1}-I_{0}\left(e^{\frac{q\left(V+I R_{S}\right)}{n k T}}-1\right)-\frac{V+I R_{S}}{R_{S}}$

where Io is the diode's saturation current, $\mathrm{q}$ is the electron's charge, $\mathrm{n}$ is the diode's ideality factor, $\mathrm{k}$ is Boltzmann's constant, T is temperature, RS is the series resistance, and RSH is the shunt resistance.

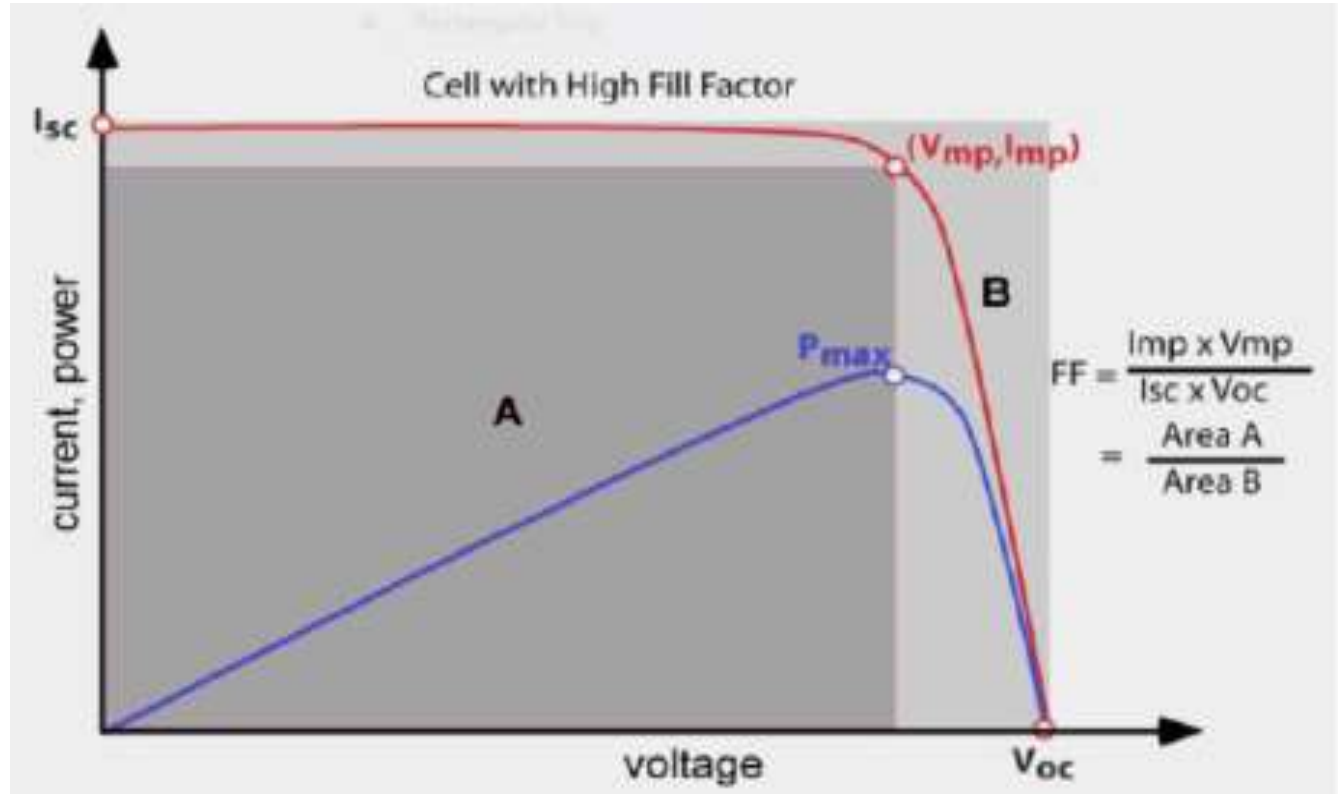

Figure 4: An I-V curve and an visual illustration of fill factor [2]. 
In single-layer organic solar cells, the value of Voc is less than the difference between the work functions of two electrodes. Experimental Voc is normally far less than the work functions difference, because free charge quantity decreases by charge recombination.

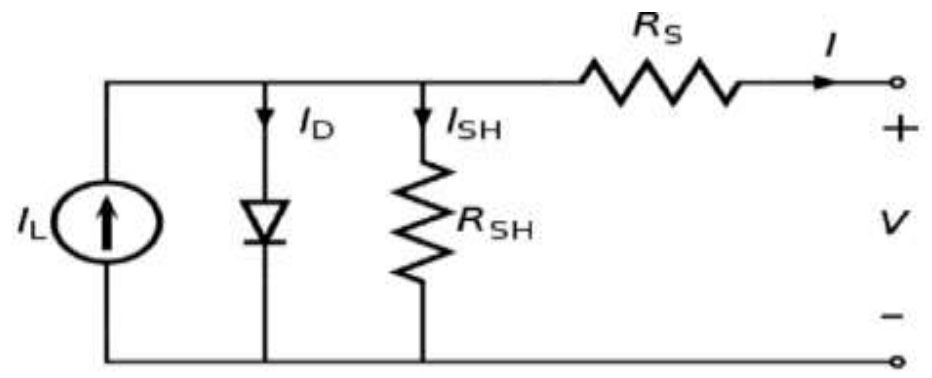

Figure 5: An equivalent circuit of a solar cell; adapted from [2].

Reducing the probability of charge recombination can improve Voc close to the value. Because lower mobility of organic materials results in higher resistance, the fill factor of single-layer photovoltaic devices is generally smaller. In hetero junction devices fabricated by the donor and acceptor, Voc depends on the LUMO level of the acceptor and the HOMO level of the donor, as well as the interface contact of the active material and electrode (Hoppe and Sariciftci, 2003). Photocurrent is linear or sub linear with intensity of absorbed sunlight (Sariciftci et al., 1993; Peumans et al., 2003). Efficiencies of OPV devices are the most important parameter for device evaluation. There are three methods to express efficiency: 1) external quantum efficiency ( $\eta \mathrm{EQE})$, or termed as incident photon to current efficiency (IPCE); 2) internal quantum efficiency ( $\eta \mathrm{IQE})$, or referred to as absorbed photon to current efficiency (APCE); 3) total conversion efficiency $(\eta)$, called power conversion efficiency ( $\eta p$ or PCE). The external quantum efficiency $\eta E Q E$ is defined as the ratio of the number of electrons collected by photovoltaic cells to the number of incident photons. In photovoltaic cells based on exciton dissociation mechanism at donor acceptor interface, $\eta E Q E$ can be expressed as,

$\eta \mathrm{EQE}=\eta \mathrm{A} \eta \mathrm{IQE}=\eta \mathrm{A} \eta \mathrm{ED} \eta \mathrm{Dis} \eta \mathrm{CT} \eta \mathrm{CC}$

Where, $\eta \mathrm{IQE}$ is the internal quantum efficiency defined as the ratio of the number of carriers collected at an electrode to the number of photons absorbed in the device, $\eta \mathrm{A}$ is photon absorption efficiency, $\eta E D$ is exciton diffusion efficiency, $\eta D$ is exciton dissociation efficiency, $\eta \mathrm{CT}$ is free charge transport efficiency, and $\eta C C$ is charge collection efficiency. If incident light is monochromatic illumination at wavelength $\lambda(\mathrm{nm}), \eta \mathrm{EQE}$ of solar cells can be rewritten as,

$\eta_{\mathrm{EQE}}=\frac{1240 \cdot J S C}{\lambda . P i n}$.

Where, Jsc is the short circuit current density with unit of $\mathrm{mAcm}-2$, Pin is the incident power with unit of $\mathrm{mW} \mathrm{cm}-2$. The internal quantum efficiency $\eta \mathrm{IQE}$ can be expressed by device absorption spectrum and external quantum efficiency as,

$\eta_{1 Q E}=\frac{\eta_{E Q E}}{1-T-R}$

Where, $T$ and $R$ are transmissivity and reflectivity, respectively. $\eta I Q E$ reveals the degree of electrical conversion from light absorbed in the device. The power conversion efficiency $\eta p$ is defined as the ratio of the maximum output power Pm to the incident power Pin, 
$\eta_{p}=\frac{P_{m}}{P_{\text {in }}}=\frac{V_{o c} J_{s c} F F}{P_{\text {in }}}$
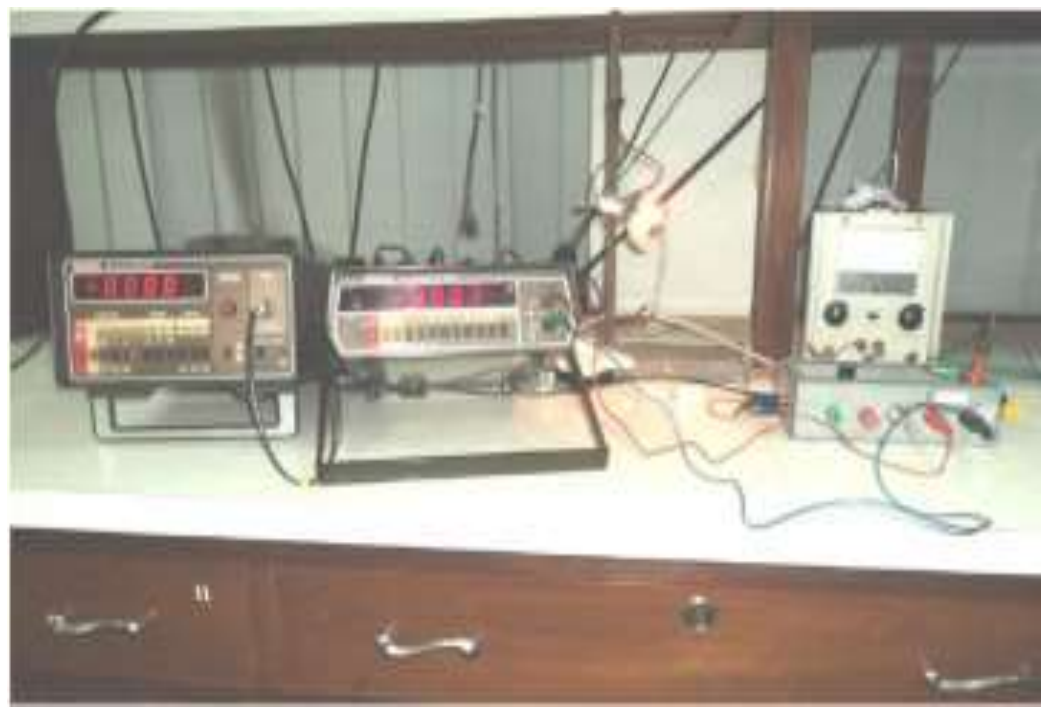

Figure 6: Experimental setup for electrical characterization

Electrical measurements were conducted in lateral direction by using 2 point probe at ambient condition. Two Keithly multi meter was used for I-V measurement and a hallozen bulb was used to irradiate the sample. Experimental set-up for electrical characterization is shown in Figure 6.

\section{Materials ANd Methods}

\section{Materials}

Graphite flakes and $\mathrm{KMnO} 4$ were purchased from Alfa Aesarand Kanto Chemical, respectively. PTFE membrane filter with a $0.45 \mu \mathrm{m}$ pore size was purchased from Millipore. Polyester hollow fiber (Tetoron, $90 \mathrm{dtex}, 38 \mathrm{~mm}$ ) was purchased from Teijin Fibers.

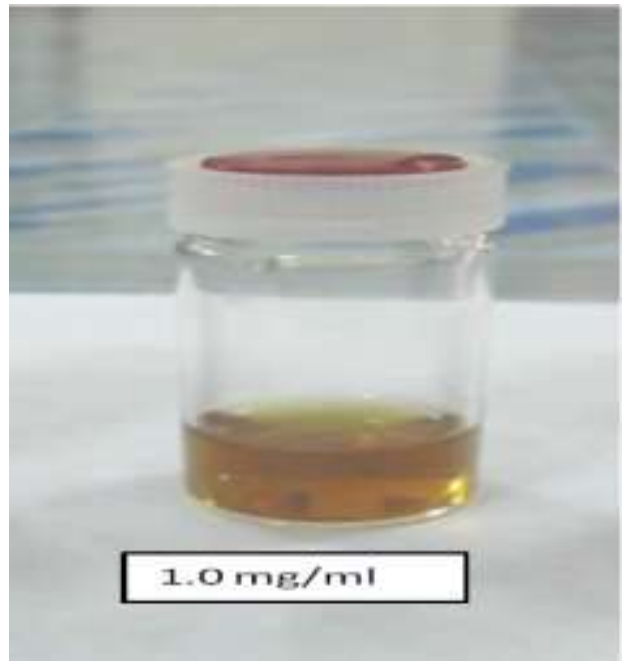

Figure 7: Photographic image of GO dispersion in water. 


\section{Synthesis of GO}

Graphene oxide was synthesized with Marcano's improved method (Cook et al., 2009). A mixture of concentrated H2SO4/H3PO4 $(180 \mathrm{~mL} / 20 \mathrm{~mL})$ was added to a mixture of graphite flakes $(1.5 \mathrm{~g})$ and $\mathrm{KMnO} 4(9.0 \mathrm{~g})$. The mixture was heated with stirring at $50{ }^{\circ} \mathrm{C}$ for $14 \mathrm{~h}$, which then was cooled to room temperature and poured onto ice (200 mL), which had been treated with $30 \% \mathrm{H} 2 \mathrm{O} 2(1.5 \mathrm{~mL})$, with further cooling in an ice bath as an exothermic reaction occurred. The mixture was filtered through polyester fiber to yield dark purple solution, which was centrifuged (3750 rpm for 5-15 h), and the supernatant was decanted away. The remaining gray solid was dispersed in water $(90 \mathrm{~mL})$ by sonication, and filtered through the polyester fiber. The filtrate was centrifuged and the supernatant decanted away. This washing protocol (dispersion by sonication, filtration, and centrifugation) was repeated with concentrated $\mathrm{HCl}(90 \mathrm{~mL})$ and ethanol $(90 \mathrm{~mL})$ then again with water, concentrated $\mathrm{HCl}$, and ethanol. The now yellow residue was coagulated by treating with diethyl ether, and filtered through a PTFE membrane to yield a yellow solid. The solid obtained on the filter was vacuum-dried overnight at room temperature, affording $0.4-0.6 \mathrm{~g}$ of dark-colored product.

\section{AFM measurements}

AFM measurements were performed by using a Dynamic Force Mode Atomic Force Microscope (SII, SPI3800N-400A) with a silicon cantilever (SI-DF3; spring constant: $1.3 \mathrm{~N} \mathrm{m-}$ 1, resonance frequency: $26 \mathrm{kHz}$ ). The frequency of the cantilever was set higher than the resonance frequency. The voltage of $1.0 \mathrm{~V}$ was applied to driven the vibration. The amplitude damping factor was determined to a default value of the apparatus. Mica and glass substrates were purchased from Nisshin EM Corporation. In order to measure the thickness of GO nanoplatelets mica substrate was used where asglass substrates used to prepare GO thin films. The mica substrates were cleaved before sample preparation. Glass substrates were cleaned in an ultrasonic bath with DI water,acetone and IPA for 15 minutes in each case. A dispersed GO/H2O solution were deposited onto the surface and dried.

\section{Result AND ANALYSIS}

The human race fretful with environmental, economic, and geopolitical consequences of energy consumption, new alternatives to traditional energy sources is needed. By rapid progress being made in organic photo voltaics, they are becoming a feasible source of renewable energy, as power conversion efficiencies (PCEs) exceeding 8\% have been confirmed. Traditional bulk hetero junction polymer solar cells consist of a transparent indium tin oxide (ITO) anode, a hole transport layer, a photoactive layer, and a top cathode. Hole transport layers must have high optical transparency, good chemical stability, a large ionization potential, and good electron blocking capability. It has already been mentioned earlier that conducting polymer, poly (3, 4-ethylenedioxythiophene): poly (styrene sulfonate) (PEDOT:PSS), has been most commonly used as an anode interfacial layer (AIL) to improve anode and to increase hole collection in organic electronics. However, PEDOT: PSS has several problems including high acidity, hygroscopic properties, and inhomogeneous electrical properties, resulting in poor long term stability. Therefore, development of cost efficient and simply processable interfacial materials has been urgently demanded. For this purpose, a thin film of GO was recently reported as an efficient hole transport layer for high-performance OSCs. However, cell performance with GO is highly sensitive to the film thickness and roughness of GO due to its insulating property. In addition, an insulating GO film can be easily tunable by chemical and thermal treatment into semi metallic chemically reduced graphene 
to facilitate the carrier transport more efficiently. In this context solution-processable graphene-based thin films as hole transport layers (HTLs) were investigated for high performance and stable polymer solar cells. Figure 4.1 represents surface morphology of a GO thin film recorded by SEM. In the SEM image it is clearly seen that the film is wrinkle free. Besides this, folding of GO sheets is also clearly visible in the SEM image.

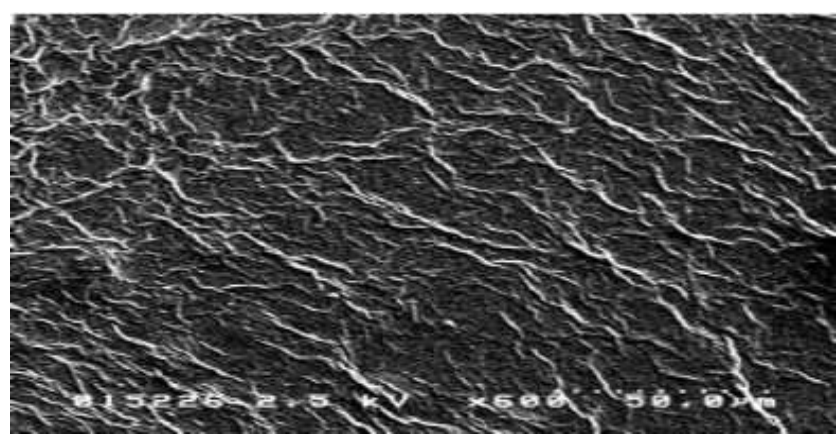

Figure 8: A low-resolution SEM image of a graphene thin film was used as HTL

Figure 9 represents a high-resolution SEM image of GO thin films which was used as HTL in OPV device. In order to measure the surface roughness of GO, AFM topography was also acquired. Figure 10 represents the typical AFM image of GO thin films. The concentration of water solution was $1 \mathrm{mg} / \mathrm{ml}$. The image reflects homogeneous deposition of the dispersed GO/H2O solution where no wrinkles and folded regions were observed on the surface. The average thickness of the film measured directly by AFM is $52.5 \mathrm{~nm}$. The film thickness far exceeds the thickness of a single layer of graphene $(\sim 1 \mathrm{~nm})$, indicating that the films prepared here mostly consist of multiple layers of GO.

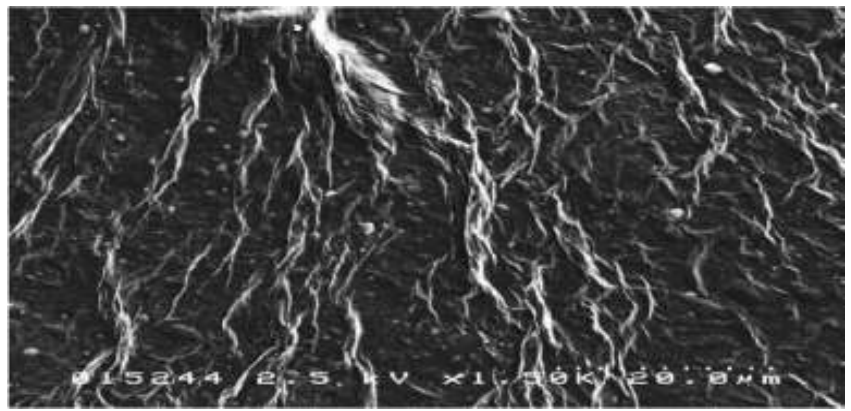

Figure 9: A high- resolution SEM image of a graphene thin film was used as HTL

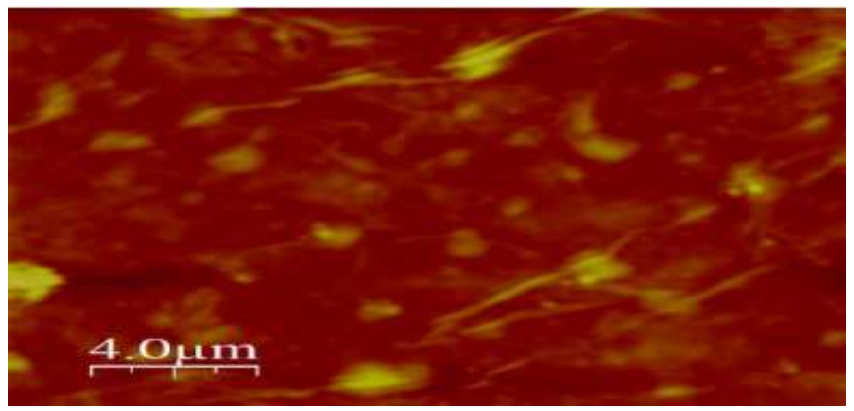

Figure 10: Typical AFM image of a graphene thin film was used as HTL 


\section{Characterizations of OPV-1}

The photovoltaic characteristics of the fabricated device (ITO/GO/P3HT:PCBM/Al) were characterized under halogen light illumination at $100 \mathrm{~mW} / \mathrm{cm} 2$. Here we have demonstrated results for two cells. The Voltage-Intensity plot for cell-1is demonstrated in Figure 11.

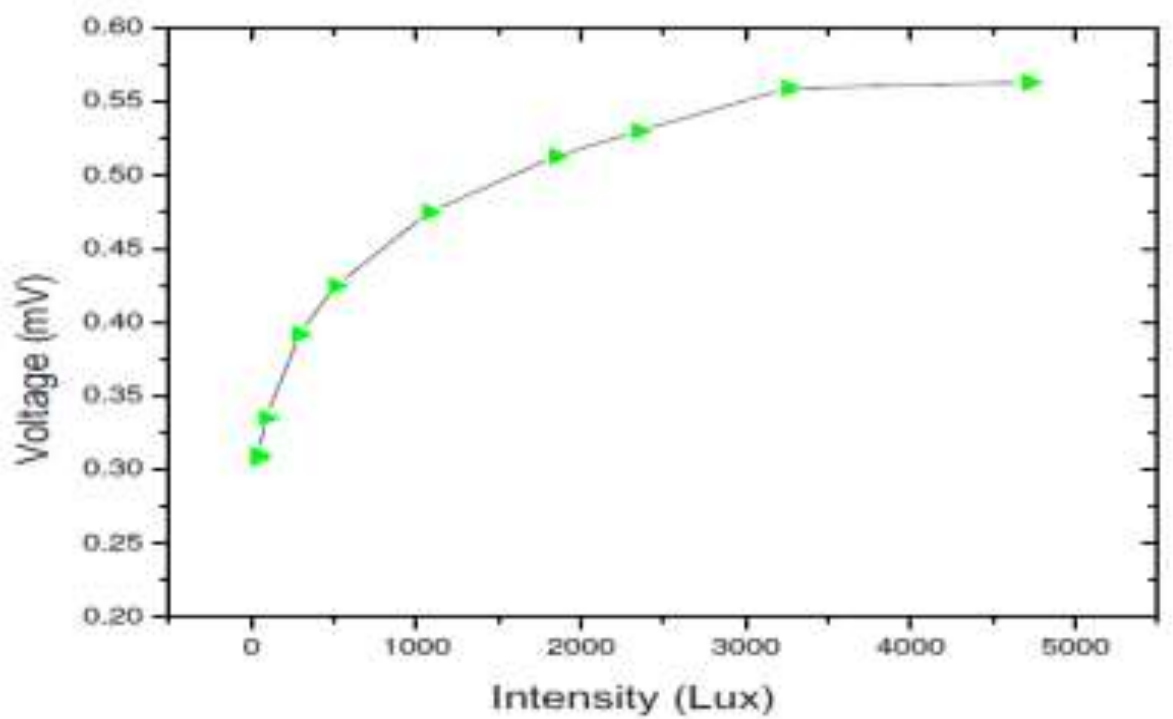

Figure 11: Cell Output voltage as a function of irradiated Halogen light intensity

The linear dependence of Resistance vs. Voltage in solar cell-1 is presented in Figure 12.

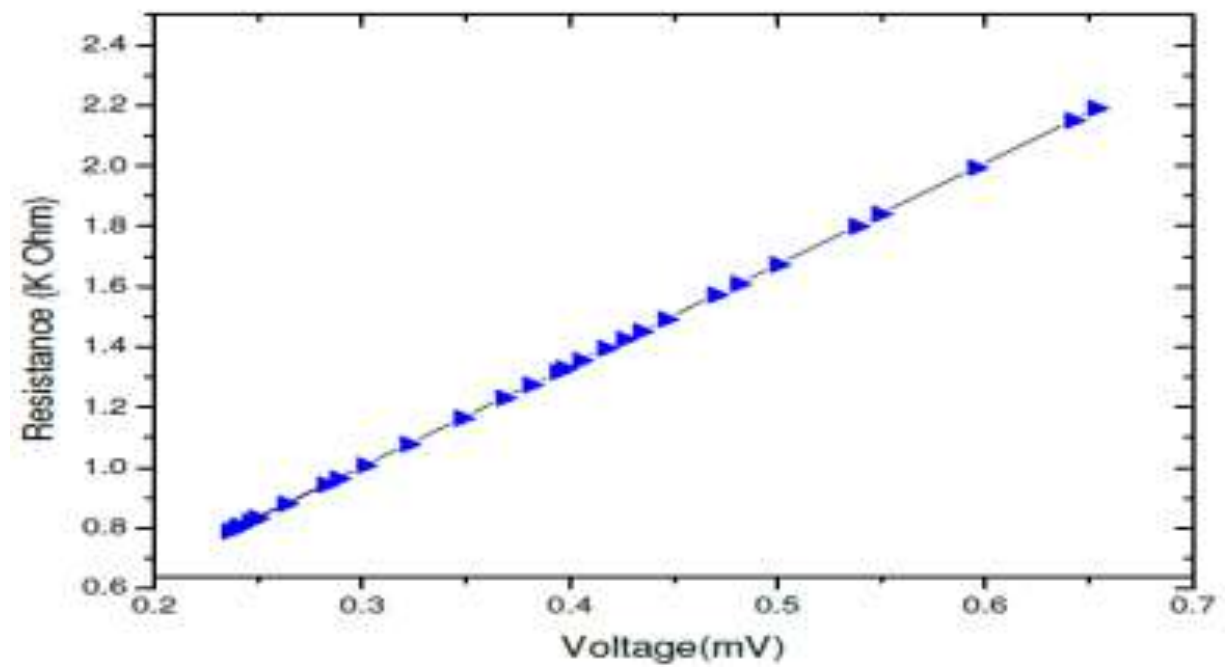

Figure 12: Resistance (K-Ohm) vs. Voltage (mV) Plot

The voltage of the device increases with the increment of the intensity of the halogen bulb. We have noticed a small amount of voltage at zero intensity (halogen) because of the normal light of the room. Initially (at 0-1000 lux) the voltage increases gradually but at a certain stage when intensities are higher like 4000-5000 lux the function almost becomes a straight line. 


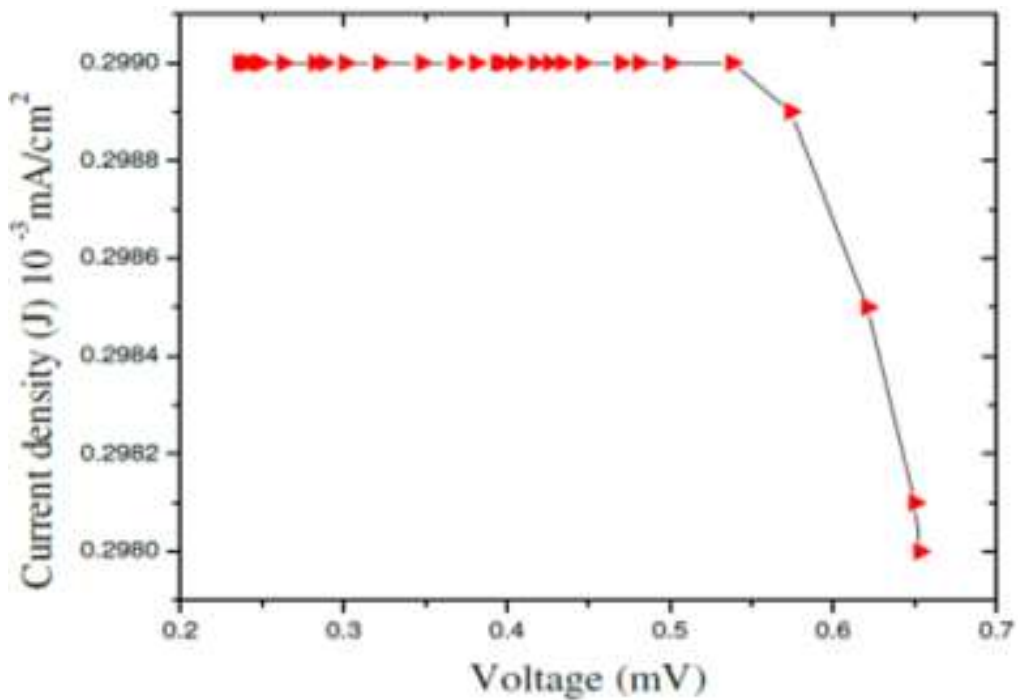

Figure 13: Current (mA) vs. Voltage $(\mathrm{mV})$ Plot

The current as a function of voltage of the devices are shown in Figure 13. The current retains the same value for the value of voltage up to $\sim 0.537 \mathrm{mV}$. Then there is a sudden fall in the current at $0.538 \mathrm{mV}$. Here the maximum output voltage is $0.538 \mathrm{mV}$.

Calculations of OPV-1 parameters:

Here,

Area of the each cell is, $\mathrm{A}=2 \times 2 \mathrm{~mm}^{2}$

Maximum current, $\mathrm{Im}_{\mathrm{m}}=0.299 \times 10^{-3} \mathrm{~mA}$

Short circuit current, Isc $=0.299 \times 10^{-3} \mathrm{~mA}$

Maximum voltage, $\mathrm{V}_{\mathrm{m}}=0.538 \mathrm{mV}$

Open circuit voltage, $\mathrm{VOC}=0.665 \mathrm{mV}$

Now, the Fill factor is,

$\mathrm{FF}=\frac{I_{\max } V_{\max }}{I_{S C} V_{O C}}$

$\mathrm{FF}=\frac{0.299 \times 10^{-3} \mathrm{~mA} \times 0.538 \mathrm{mV}}{0.299 \times 10^{-3} \mathrm{~mA} \times 0.665 \mathrm{mV}}=0.809$

We know, the power conversion efficiency is

$\eta_{p}=\frac{P_{m}}{P_{\text {in }}}=\frac{V_{O C} J_{S C} F F}{P_{\text {in }}}$

Where,

Current density, $\mathrm{J}_{\mathrm{sc}}=\frac{I s c}{A} \mathrm{~mA} / \mathrm{cm}^{2}=\frac{0.299 \times 10^{-3} \mathrm{~mA}}{2 \times 2 \times 10^{-2} \mathrm{~cm}^{2}}=7.475 \times 10-3 \mathrm{~mA} / \mathrm{cm}^{2}$

Incident power, $\mathrm{P}_{\text {in }}=100 \mathrm{~mW} / \mathrm{cm}^{2}$ (with AM 1.5); when the distance between the sample and the light source is $=14 \mathrm{~cm}$

So, the efficiency is, $\eta_{p}=\frac{0.665 \mathrm{mV} \times 7.475 \times 10^{-3} \mathrm{~mA} / \mathrm{cm}^{2} \times 0.809}{2 \times 2 \times 10^{-2} \mathrm{~cm}^{2}}$

The average short circuit current density (Jsc), open-circuit voltage (Voc), fill factor (FF), and power conversion efficiency values for OPV- 1 is $0.007475 \mathrm{~mA} / \mathrm{cm} 2,0.665 \mathrm{mV}, 0.809$, and $0.00421 \%$ respectively. 


\section{Characterizations of OPV-2}

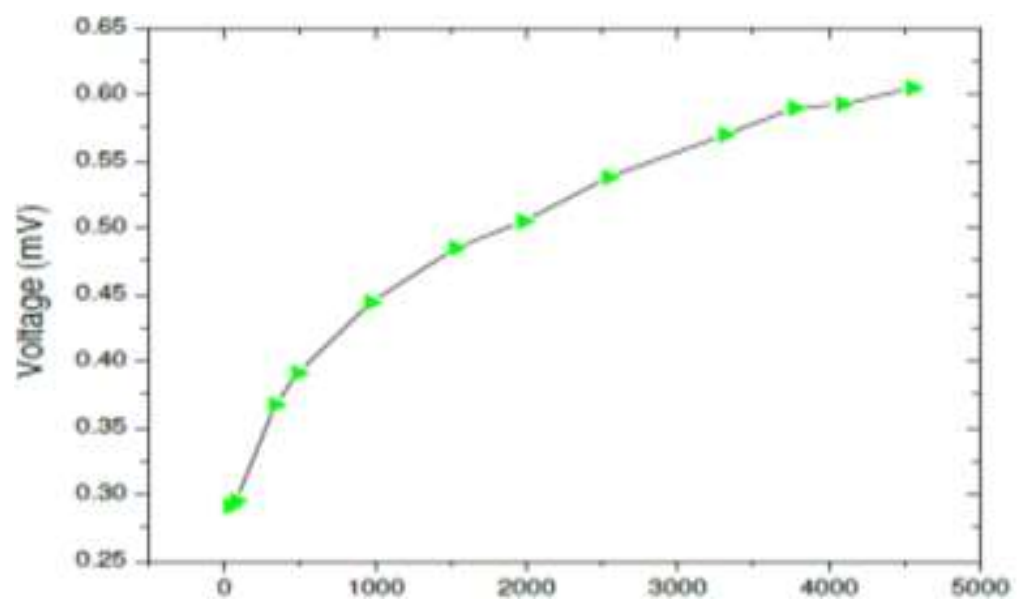

Figure 14: Cell Output voltages a function of irradiated Halogen light intensity

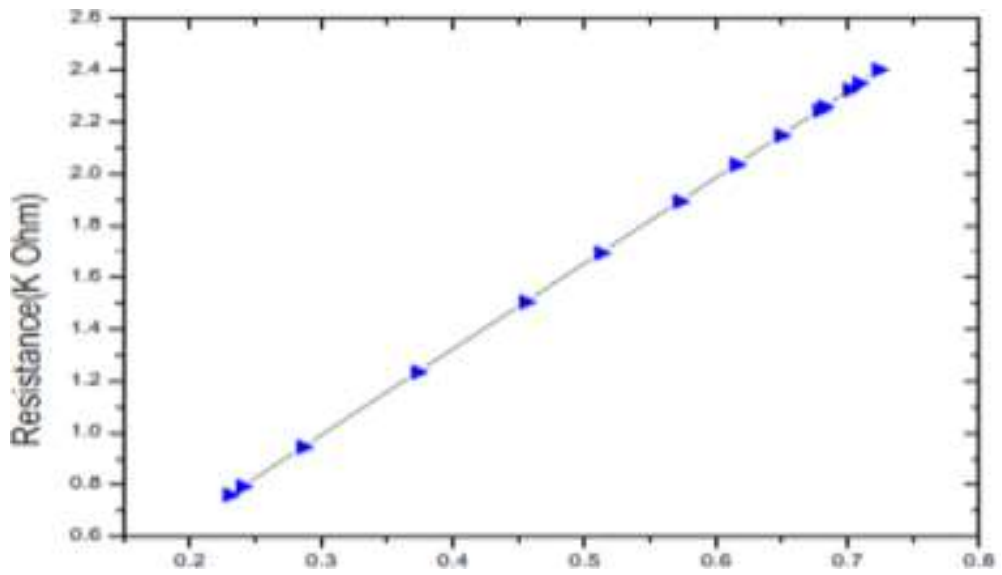

Figure 15: Resistance vs. Voltage (mV) Plot

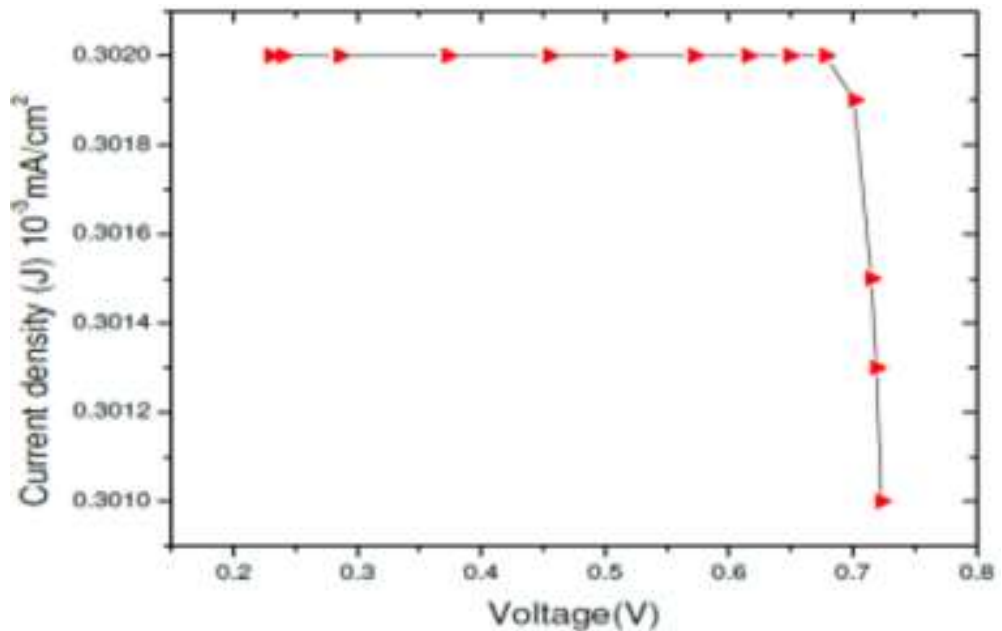

Figure 16: Current (mA) vs. Voltage (mV) Plot 
Calculations of OPV-2 parameters:

Here,

Area of the each cell is, $A=2 \times 2 \mathrm{~mm}^{2}$

Maximum current, $I_{m}=0.302 \mu A=0.302 \times 10^{-3} \mathrm{~mA}$

Short circuit current, $I_{s c}=0.302 \mu A=0.302 \times 10^{-3} \mathrm{~mA}$

Maximum voltage, $V_{m}=0.678 \mathrm{mV}$

Open circuit voltage, $\mathrm{VOC}=0.725 \mathrm{mV}$

Now, the Fill factor is,

$F F=\frac{I_{\max } V_{\max }}{I_{s c} V_{o c}}$

We know, the power conversion efficiency is,

$\eta_{p}=\frac{P_{m}}{P_{\text {in }}}=\frac{V_{o c} J_{s c} F F}{P_{\text {in }}}$

Where,

Current density,

$J_{s c}=\frac{I_{S c}}{A} m A / \mathrm{cm}^{2}=\frac{0.302 \times 10^{-3} \mathrm{~mA}}{2 \times 2 \times 10^{-2} \mathrm{~cm}^{2}}=7.55 \times 10^{-3} \mathrm{~mA} / \mathrm{cm}^{2}$

Incident power, $P_{\text {in }}=100 \mathrm{~mW} / \mathrm{cm}^{2}$ (with AM 1.5); distance of the sample and the lightsource $=14 \mathrm{~cm}$

So, the efficiency is,

$\eta_{p}=\frac{0.725 \mathrm{mV} \times 7.55 \times 10^{-3} \mathrm{~mA} / \mathrm{cm}^{2} \times 0.935}{100 \mathrm{~mW} / \mathrm{cm}^{2}}=0.05117 \times 10^{-3}=0.0051 \%$

The average short circuit current density (Jsc), open-circuit voltage (Voc), fill factor (FF), and power conversion efficiency values for OPV-2 is $0.00755 \mathrm{~mA} / \mathrm{cm} 2,0.725 \mathrm{mV}, 0.935$ and $0.0051 \%$ respectively. Comparing these results to the literature is complicated by the fact that these cell are fabricated outside of glove boxes. In addition, very often cells in the literature are immediately encapsulated, and the amount of data about the effect of encapsulation in this thesis is limited.

It's possible that the curing process, which required that the cell sit under a UV lamp for several minutes, is responsible for the quick deterioration. Painting the edges of a small piece of glass and pressing the glass over the active material would prevent UV light from damaging the cell. This process should be considered in future encapsulation tests. It's also possible that oxygen and water that discussed into the cell before encapsulation occurred was responsible for the cell's degradation. The unencapsulated cell was tested under ambient conditions with constant illumination by a metal-halide lamp at 1 sun.

This cell degraded to $60 \%$ of its initial efficiency after approximately 50 hours. The inverted cells were significantly more stable than the normal OPVs. Unfortunately, the cells were not tested through the night or the following morning, so crucial information about the lifetime of the cells was not recorded. In future experiments, a Lab View program that automatically takes data at regular intervals should be developed in order to track OPV performance over many hours or days. White et al. (2006) took preliminary device lifetime measurements, which are summarized in Table 
Table 1: Degradation of (White et al., 2006) inverted OPV (one sun of simulated AM1.5 illumination)

\begin{tabular}{|l|l|l|}
\hline DAY & PCE (\%) & Percent of Initial PCE (\%) \\
\hline 1 & 2.97 & 100 \\
\hline 4 & 2.58 & 87 \\
\hline 7 & 2.3 & 2 \\
\hline
\end{tabular}

Unlike the cells tested in this thesis, the cells in White's study were stored in nitrogen glove boxes between measurements. The results from this paper indicate that unencapsulated, inverted P3HT: PCBM solar cells can last for over a week if stored in inert environments. White et al. note that device performance improved when the $\mathrm{ZnO}$ and silver layers were exposed to air; however, the active material degraded under ambient conditions, rendering neither ambient nor inert storage ideal for cell stability.

\section{CONCLUSION}

We demonstrated that solution-processable graphene-based thin films can be used as an efficient anode interfacial layer for high-efficiency and high-stability OSCs. Conventional rGO was investigated for use as an efficient interfacial layer for high performance OSCs. In particular, dramatically enhanced performance of organic solar cells was demonstrated by introducing r-GO as an AIL into the OSCs, and the overall photovoltaic characteristics. This thesis met its primary objective by successful demonstrating processes to synthesize and characterize P3HT: PCBM solar cells. The efficiencies of the two normal P3HT: PCBM solar cells, $0.00421 \%$ and $0.0051 \%$ respectively, were modest compared to high-performing inorganic photo voltaics, but not drastically different from the efficiencies of P3HT: PCBM OPVs in the literature. It is expected that the efficiencies would be better if the device were kept in a vacuum globe box. Like most organic solar cells, device lifetimes were short. The normal cell illuminated by the tungsten halogen lamp at an intensity of $530 \mathrm{~W} / \mathrm{m} 2$ degraded of its initial efficiency day by day. Since organic solar cells hold great promise as a source of low cost, clean energy, the research conducted in this thesis should be continued and improved. More data and tests of the effect of encapsulation on device lifetimes are needed. In addition, inverted cells should be fabricated and their stabilities should be monitored over several days. To get higher yields for normal and inverted devices, a more meticulous approach at understanding the reasons for OPV failure should be taken. In the future, a better lamp, one that is calibrated and has a spectrum that more closely matches the solar spectrum, would greatly improve the characterization process and would make it easier to compare results to values reported in the literature. Finally, synthesizing an OPV with a CVD graphene electrode would be a significant accomplishment and should be attempted in future work. Graphene has a variety of advantages over ITO, and replacing ITO with graphene may help OPVs attain commercial viability in the future. A practical replacement for conventional PEDOT: PSS, thus further advancing the realization of high-efficiency, high stability, and low-cost OSC sy high-throughput roll-to-roll manufacturing.

\section{REFERENCES}

Cook S., Katoh R., Furube A., Phys. Chem. 113 (6), 2009, 2547-2552.

Hoppe H., Sariciftci N. S., J Mater Res.19, 2004, 1924-1945.

National Instruments. Part ii-photovoltaic cell i-v characterization theory and labview analysis code. Development Zone, URL:http://zone.ni.com/devzone/cda/tut/p/id/7230.

Peumans P., Yakimov A., Forrest S. R., Appl Phys.93, 2003, 3693-3723. 
Sariciftci N. S., Braun D., Zhang C., Srdanov V. I., Heeger A. J., Stucky G., Wudl F., Appl Phys Lett. 62, 1993, 585-587.

Wang Y., Gu P., Cao J., Lv T., Zhang T., Wang Y., Zhang Y., Advanced Materials Research 468, 2012, 1823.

White M. S., Olson D. C., Shaheen S. E., Kopidakis N., and Ginley D. S., Applied Physics Letters, 89(14): $143517\{143517\{3$, October 2006.

$$
--0--
$$

ISSN: $2409-3629$

Online Archive Link: https://abc.us.org/ojs/index.php/ei/issue/archive 